\title{
Bacterial quorum sensing allows graded responses to variations in density, on both individual and population scales
}

\author{
Jennifer Rattray ${ }^{1,2}$, Stephen Thomas ${ }^{1,2,3}$, Yifei Wang ${ }^{1,2,4}$, Sam P. Brown ${ }^{1,2}$ \\ ${ }^{1}$ School of Biological Sciences, Georgia Institute of Technology, Atlanta, 30332 GA, USA \\ ${ }^{2}$ Center for Microbial Dynamics and Infection, Georgia Institute of Technology, Atlanta, 30332 GA, USA \\ ${ }^{3}$ Graduate Program in Quantitative Biosciences (QBioS), Georgia Institute of Technology, Atlanta, 30332 \\ GA, USA \\ ${ }^{4}$ The Institute for Data Engineering and Science (IDEaS), Georgia Institute of Technology, Atlanta, 30332 \\ GA, USA
}

\begin{abstract}
Quorum sensing (QS) is a mechanism of cell-to-cell communication via diffusible signal molecules that controls multiple secreted factors including virulence factors in bacterial pathogens $[1,2]$. While the standard view is that QS functions as a density-sensing mechanism, the functional and evolutionary context of QS continues to be disputed [311]. A critical step in assessing the various adaptive hypotheses is establishing the functional capacities and limits of QS. Current functional studies largely focus on a dichotomy of QS on/off (or, quorate / sub-quorate) states, despite the increasing amount of heterogeneity on a cellular scale [4,12-16], overlooking the potential for intermediate, graded responses. In this paper we explore the functional capacity of QS to resolve finely graded environmental densities and introduce the use of reaction norms as a way to holistically characterize QS response. Here we show that Pseudomonas aeruginosa can deliver a graded response to variation in environmental population density on both the population and individual scales. We further resolve the linear population response to be the product of two component cellular reaction norms: the likelihood of being responsive and the intensity of response. Overall, this work reveals that there is no critical cell mass or 'quorum', at which QS is activated on either the individual cell or population scale.
\end{abstract}

\section{INTRODUCTION}

Many species of bacteria are capable of a form of cell-cell communication via diffusible signal molecules, generally referred to as quorum sensing (QS). The study of QS has largely focused on the intracellular scale, leading to a detailed understanding of the regulatory mechanisms shaping the production of and response to signal molecules in model organisms such as Vibrio cholerae, Bacillus cereus and Pseudomonas aeruginosa [1]. We now understand that QS is mediated by multiple diffusible signals that together control a diverse array of responses, including swarming, luminescence, competence and the production of diverse secreted factors [17].

While the molecular mechanisms of QS have been described for model organisms in remarkable detail, the functional and evolutionary context of QS continues to be disputed. In other words, while we understand how QS works, we still have limited understanding of why bacteria use this system to control behavior. What are the functions of QS? How do these QS functions help bacteria to survive and grow? The standard answer is that 
bacteria use QS to sense when they are at sufficient density ('quorate') to efficiently turn on cooperative behaviors such as secretion of toxins and enzymes in order to collectively modify their environment [5]. Other researchers have argued that QS is an asocial sensing apparatus, where individual cells produce and monitor signal levels in order to infer their physical environment (am I in an open or enclosed space?) [18]. More recently, integration of molecular and evolutionary approaches has increased the menu of potential functions to include sensing multiple aspects of both the social and physical environment [3-6] and coordinating complex social strategies that limit the profitability of noncooperating 'cheat' strains [7-11].

A critical step in assessing the various adaptive hypotheses is establishing the functional capacities and limits of QS. Previous studies have demonstrated 'density sensing' functions -populations can use QS to sense when they exceed a density threshold $[5,19,20]$. In addition, Darch et al. demonstrated that responding with increased QS controlled cooperative activity at high density can provide a fitness benefit [5]. Other studies have demonstrated 'diffusion sensing' functions [18] -QS systems can functionally respond to variation in physical containment, so that even a single cell can become 'quorate' (turn on a QS controlled reporter gene) if isolated in a sufficiently small contained space [4]. More recently, some studies have demonstrated 'genotype sensing' functions - QS can respond to variation in the genotypic composition of a population, restricting QS-controlled responses to populations that are enriched with wildtypes $[7,8,21,22]$.

The functional studies outlined above largely focus on a dichotomy of QS on/off (or, quorate / sub-quorate) states, overlooking the potential for intermediate, graded responses (Fig 1A). The threshold quorate/non-quorate concept is ingrained in the QS literature following the use of the legal 'quorum' analogy [20], and is also supported by mathematical models of QS signal dynamics that highlight how sufficiently strong positive feedback control of signal production can produce a sharp threshold response to changes in environmental parameters such as density or diffusion $[23,24]$. However, these same mathematical models indicate that graded-responses are also possible, dependent on the model parameterization. More generally, Fig 1A highlights that the phenotypic response of QS bacteria to differing environmental conditions can be viewed as a 'reaction norm' [25-28] that can in principle take differing shapes. Reaction norms describe phenotypic responses of a single genotype (y-axis, Fig 1A) to varying environmental inputs (x-axis, Fig 1A). With this reaction norm framework, it is important to emphasize that in our study the $\mathrm{x}$-axis is not time, but instead discrete environmental conditions. Whether responses are graded or thresholded during the growth towards high density in a single colony is a separate issue. Describing the reaction-norms of QS cells and populations to environmental changes is an important step towards understanding the capacities of QS systems to differentially respond to novel environments. 


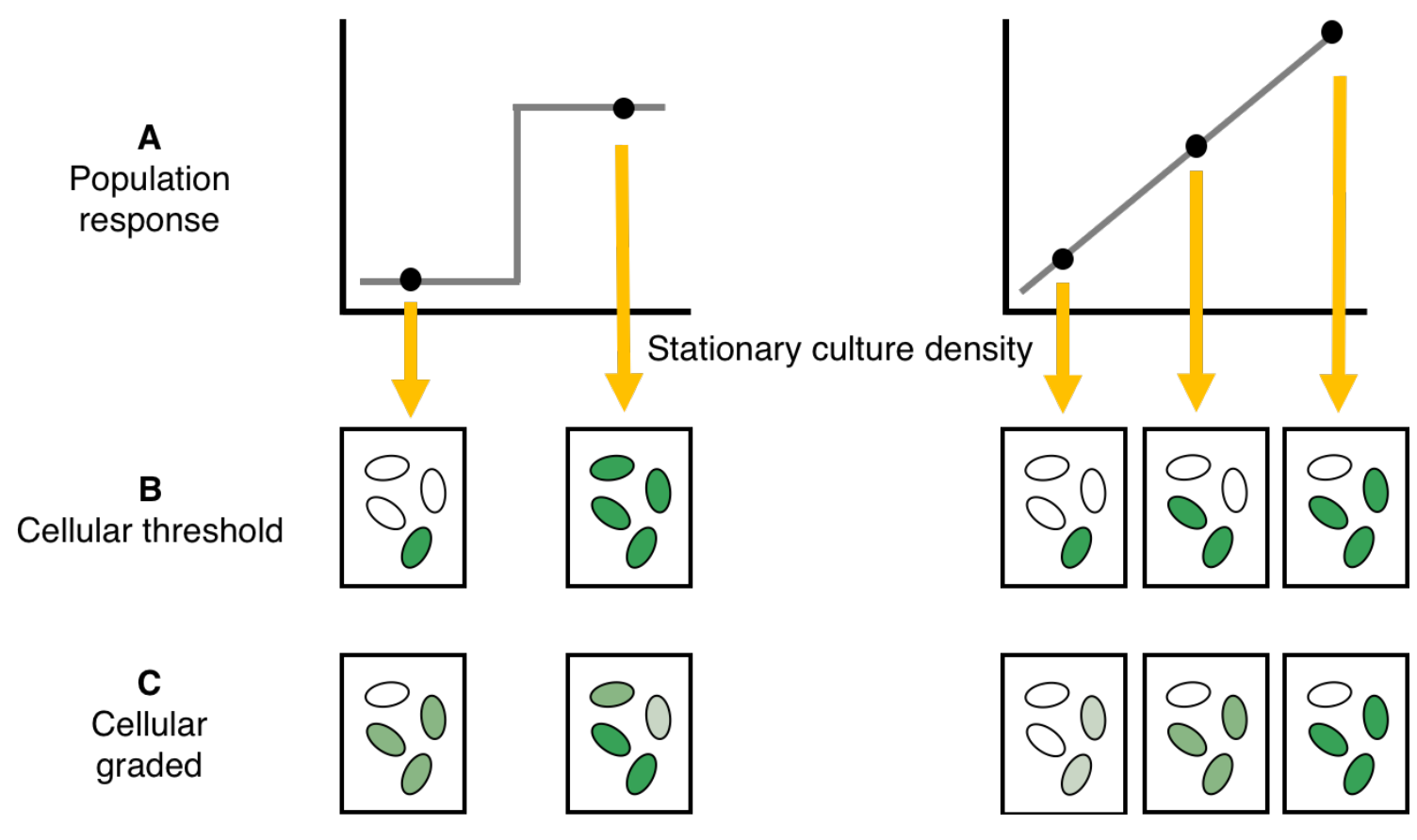

Figure 1. Schematic of potential population and single cell responses to variation in stationary phase density. A) Population response (y-axis) across discrete stationary culture density environments (x-axis), given a threshold (left) or graded response (right). In (B) and (C) we outline alternative cell-scale responses (intensity of green cells) that are consistent with discrete population-scale behaviors (yellow arrows). (B) threshold (ON/OFF) cellular responses can produce a threshold or graded responses on population scale. (C) graded individual responses can produce threshold or graded responses on a population scale.

Whether the population-scale reaction norm to environmental variation is threshold-like or graded (Fig 1A), a separate question is how collective population level responses are constructed out of individual cellular contributions (Fig 1B,C). Studies of QS on a singlecell scale have revealed substantial heterogeneity in response to QS signals $[4,12-16]$, highlighting that cell-cell communication does not necessarily result in tight synchronization of individual cell activity (Fig 1B,C). In some systems, heterogeneity can be quenched by the addition of extra signal [13,15], implying a lack of receptor saturation. However, this is not a universal result [12], indicating that other molecular processes can drive cellular variation in response. Regardless of the molecular details, we currently lack a functional understanding of how individual cellular responses vary with changes in the environment.

In the current study we address the canonical 'density sensing' function of QS, using the environmental generalist and opportunistic pathogen Pseudomonas aeruginosa, and an unprecedented scale of reaction norm resolution (17 different stationary phase densities). Our first challenge is to map the population-scale resolving power of QS to quantitatively discriminate graded differences in population density (Fig 1A). Does P. aeruginosa respond in a purely threshold manner, collapsing quantitative differences in population density into a simple low / high qualitative output, or can QS allow P. aeruginosa to deliver a graded response to distinct environmental densities? Our second challenge is to 
understand how collective responses are partitioned across individual cells. Are changes in collective responses governed primarily by changes in the proportion of cells in an on state (Fig 1B) or changes in the individual cell intensity of response (Fig 1C), or both?

\section{RESULTS}

Collective level of response to density is linear and graded. Our first challenge is to map out the population scale reaction norm of the collective QS-controlled response to variation in stationary phase population densities. To provide a detailed picture of the QS response reaction norm to varying density, we grew a QS reporter strain (PAO1 lasB$G F P$ ) under 17 conditions of carbon limitation and measured average fluorescence output per cell at early stationary phase per condition (Fig 2). The range of cell densities generated from this method is from $1 \times 10^{8}$ cells $/ \mathrm{ml}$ to $2 \times 10^{9}$ cells $/ \mathrm{ml}$.

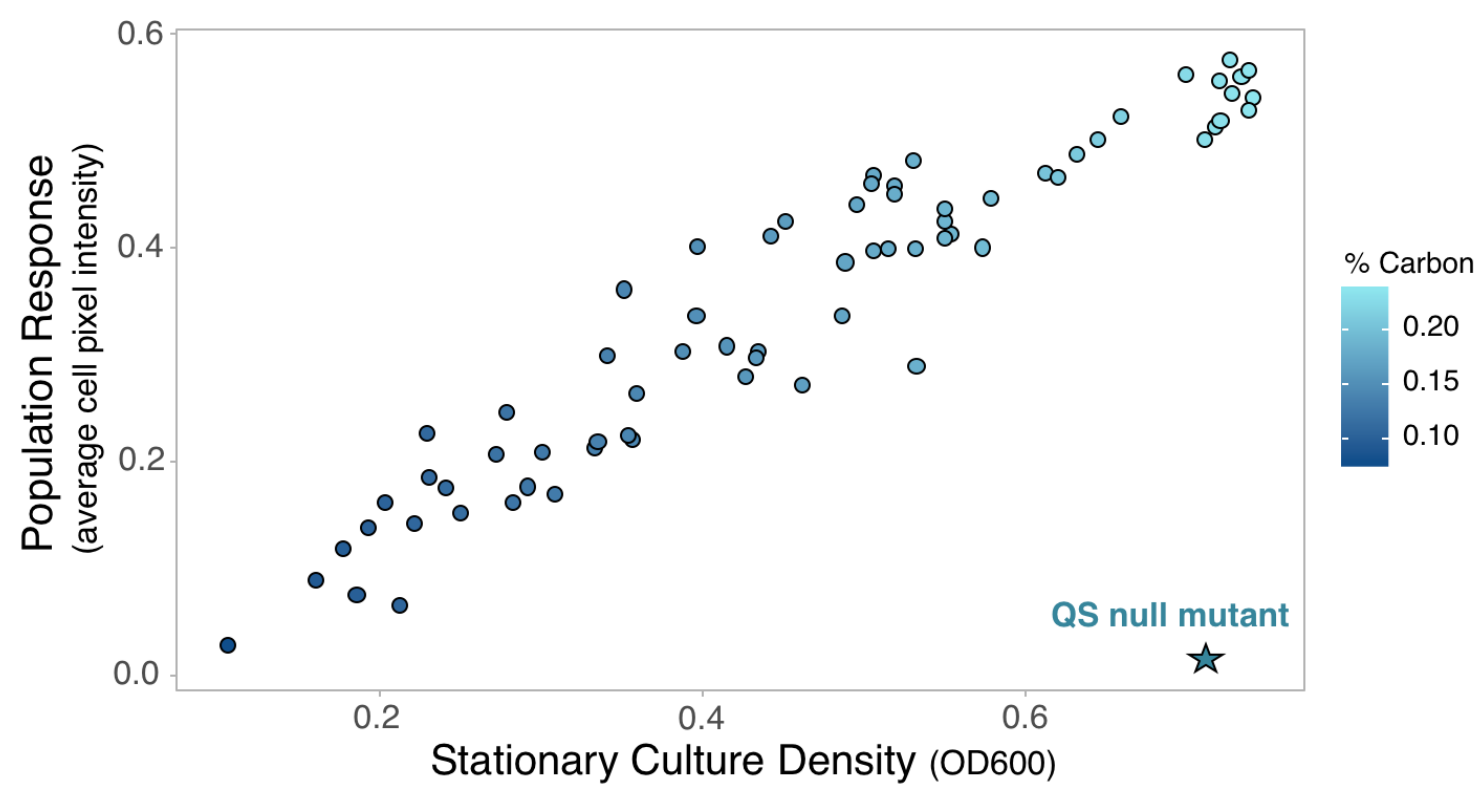

Figure 2. Population response to increasing cell density is linear and graded. Stationary culture density was controlled by manipulating the concentration of casamino acids as the limiting resource (Fig S1). Cells were grown to stationary phase and assayed for QS response via fluorescence microscopy imaging. Response is determined by a translational fusion of the quorum sensing controlled las $B$ promoter and a green fluorescent protein. Individual cell pixel intensity is a measure of quorum sensing response and average pixel intensity is calculated across all cells in the population as a proxy for total population expression. Data is congruent with microplate results (Fig S2). Quorum sensing signal knockout $(\Delta l a s I \Delta r h l l)$ shows background response (GFP) with no signal in the environment. Average population investment in QS increases as culture density increases with no observable density threshold (AIC linear model fit: 1.56, AIC step-function fit: 71.03). Range of stationary phase cell densities is from $1 \times 10^{8}$ cells $/ \mathrm{ml}$ to $2 \times 10^{9}$ cells $/ \mathrm{ml}$.

Figure 2 shows that QS response is linear with increasing culture density, providing intermediate levels of per-capita response to intermediate densities. To confirm the lack of threshold behavior we assessed alternate statistical models including threshold functions, and found that a linear fit model supports the data significantly more than a 
step-function fit (AIC linear: 1.56, AIC step-function: 71.03), supporting a graded population response as outlined in Figure 1.

Individual response to density is bimodal. Figure 2 establishes that on a collective scale, the response to environmental variation (in stationary density) is smoothly graded. Next, we ask how this collective response is built from individual cell contributions. Is the graded increase due to more cells turning on at higher densities (Fig 1B), cells turning on to a greater extent (Fig 1C) or both? To address this question, we take the same data presented in Figure 2 and now present the distribution of individual cellular responses rather than simply the mean response (Fig 3).

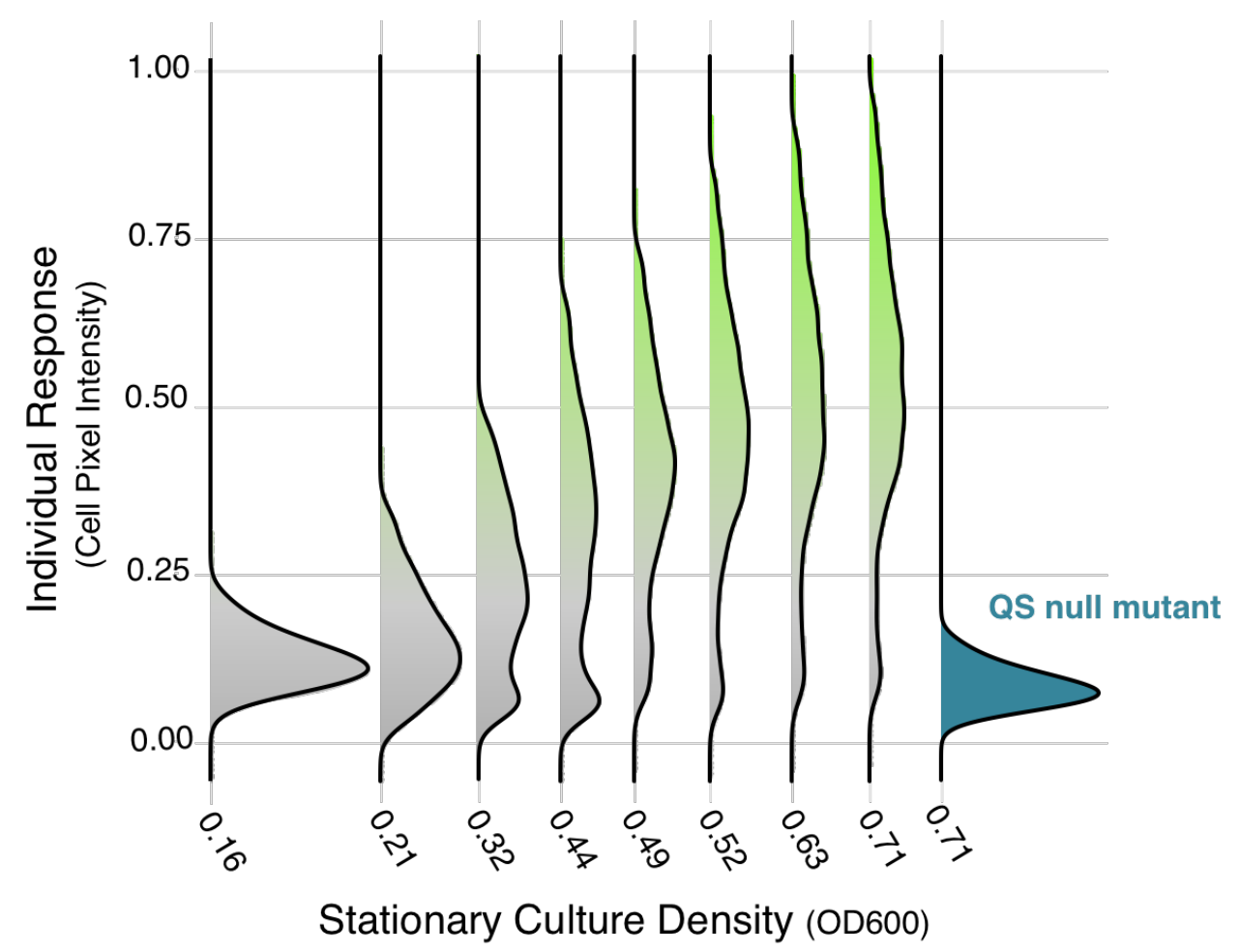

Figure 3. Individual response is bimodal. Ridgeline plot of cellular response data showing the distribution of cellular expression across the population. Populations exhibit a non-uniform response to increasing density. The lowest population density matches the QS null mutant (quorum sensing signal knockout, $\Delta l a s I \Delta r h l I$, in blue on the far right). Populations with higher cell densities split into two subpopulations, unresponsive and responsive. A total of 55 populations were sampled across 17 stationary phase environments. Representative samples were chosen for this plot by selecting populations with $\sim 0.1$ OD600 increments. Between 5,000 and 15,000 individual cells were measured per population.

As expected from prior studies [4,12-16], plotting all individual responses within a population shows cell-to-cell variation in QS response within a single population despite isogenic and homogenous culture conditions (Fig 3). At higher densities we see evidence of a bimodal distribution, with the population segregating into an unresponsive, subquorate, OFF state and a responsive, quorate, ON state. Looking across populations grown to differing stationary phase densities, we see qualitative evidence of a graded shift in the ON state towards higher cellular intensities at higher densities. To further analyze the single-cell scale data in Fig 3 we fitted a mixed normal statistical model to 
the data (Fig 4A), which allows us to define the number of cells in the OFF or ON states (Fig 4B), and also the average intensity of the ON state (Fig 4C).

A

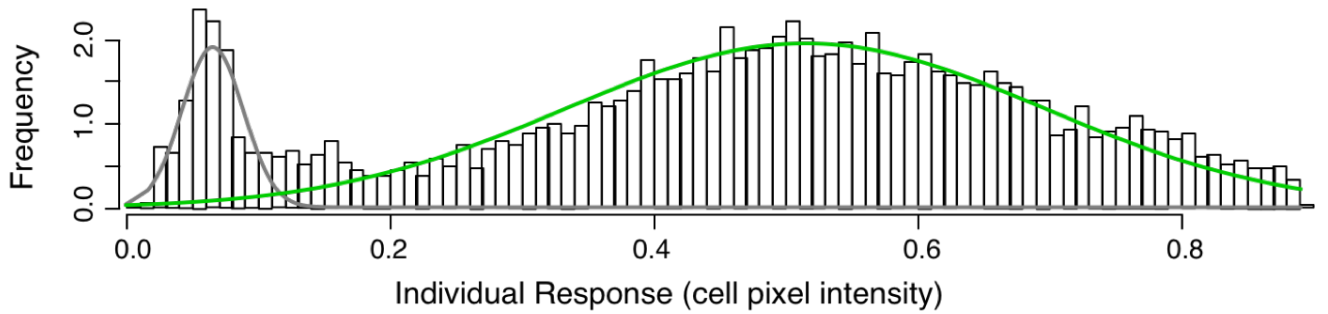

B

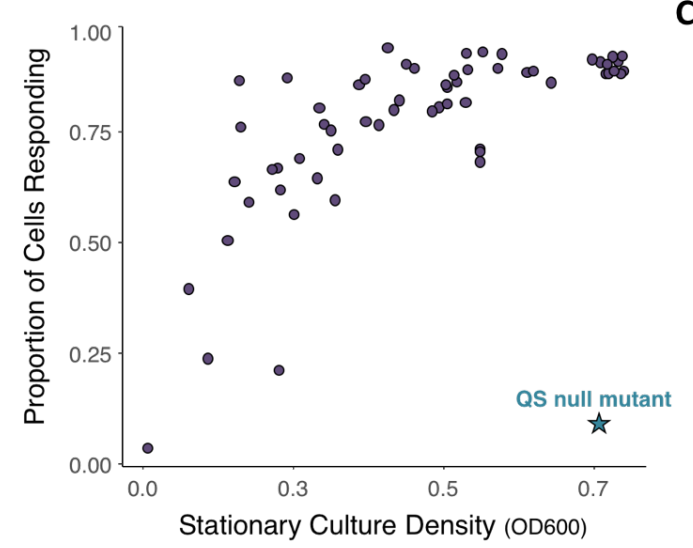

C

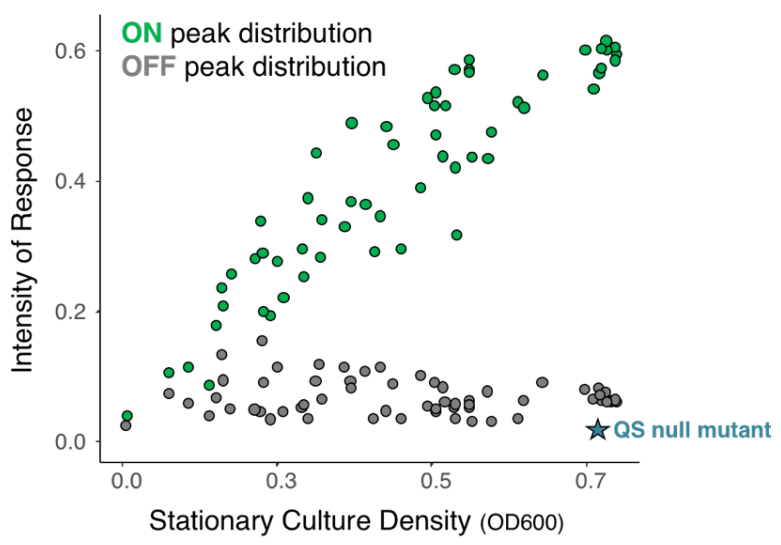

Figure 4. Proportion of cells responding and level of response varies with density. In light of the binomial responses (Fig 3), we course-grain the data into discrete states, and further quantify the magnitude of the ON state. A) Method summary. By fitting two gaussian distributions (grey and green curves) via the mixtools package in R, mixing proportions (proportion of total individuals that fall under each distribution) and mean of each distribution can be quantified. Cells in the left, grey distribution are defined as OFF, shorthand for a sub-quorate, unresponsive state which is indistinguishable from the QS null mutant (Fig 3). Cells in the right, green distribution are defined as ON, shorthand for a quorate, responsive state. The histogram shows the distribution of cellular expression levels in a single density treatment (0.42 OD600). B) Proportion of cells ON in the population as determined from the mixing proportions of a Gaussian fit mixed model. Proportion of cells ON increases with culture density but does not reach 100\%. C) Level of response as determined from the means of the fit distributions. The mean intensity of the ON state distribution increases as culture density increases, while the mean of the OFF state remains constant.

Figure 4B illustrates persistent cellular heterogeneity across the range of assayed environments, with the proportion of responding $(\mathrm{ON})$ cells increasing rapidly from 0.03 towards a plateau of 0.95 responsive cells at higher densities. In addition to the variation in proportion responding, the responsive $\mathrm{ON}$ proportion linearly increases their per-cell investment (Fig 4C). In other words, low density environments are characterized by low frequencies of 'ON' cells that invest at a low level, whereas high density environments are characterized by near universal ' $O N$ ' states with high levels of per-cell investment. Our analysis in Figure 4B,C depends on a course-graining of the underlying data (Fig 3) into a simple 2-parameter summary (proportion $\mathrm{ON}$, intensity $\mathrm{ON}$ ), via mixed normal model fits (Fig 4A). To assess the validity of the statistical summaries in Figure 4, we contrast the predicted mean intensity per population (the product of proportion ON times intensity $\mathrm{ON}, 4 \mathrm{~B} * 4 \mathrm{C}$ ) with the observed mean intensity per population (Fig 1). The agreement between observed and expected values (Fig 5; mean absolute percentage error 
$=9.7 \%$ ) indicates that the data is adequately described by a simple decomposition into proportion $\mathrm{ON}$ and intensity $\mathrm{ON}$.

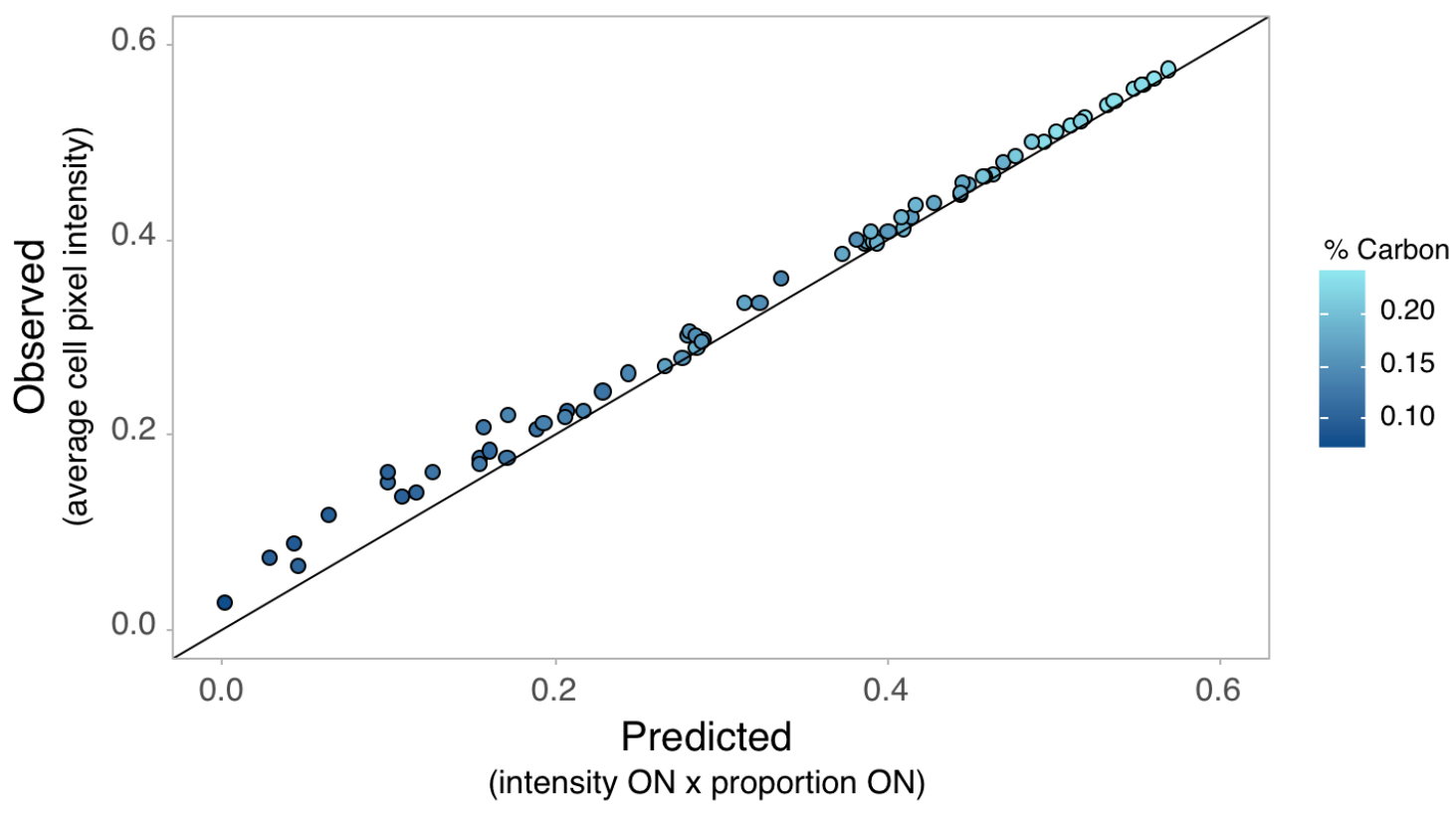

Figure 5. Proportion of cells responding and mean level of response explain population level response. Plotting the observed average pixel intensity data (Fig 2) against the predicted response (intensity $\mathrm{ON} x$ proportion cells $\mathrm{ON}$, Fig 4B,C) indicates that the two predictor parameters capture the observed population response (mean absolute percent error $=9.7 \%$ ).

\section{DISCUSSION}

Our results show that populations of $P$. aeruginosa can respond in a graded manner to variation in stationary phase density (Fig 2), and that this population-scale graded response is due to both a graded increase in the number of responsive ' $O N$ ' cells and the intensity of the 'ON' state (Figs 3-5). The ability to achieve a graded population-scale response implies in principle that $P$. aeruginosa can tune collective responses (such as the secreted elastase protein produced by our las $B$ reporter gene) to graded environmental changes, rather than simply course-graining into a simple 'high / low' dichotomy. A similar population scale graded response to continuous environmental variation was reported by Allen et al., looking at variation in the genotypic concentration of mixed populations grown to the same total density [7]. As the proportion of wildtype (PAO1 versus $\Delta$ las $B$ 'cheats') increased, the wildtype per-capita investment in cooperative LasB secretions also increased, providing a simple behavioral mechanism to protect cooperative investments from exploitation by cheats $[7,22]$. Darch et al. also reported a graded response in $l a s B$ expression across 5 density environments, all of which fall into the lower $3^{\text {rd }}$ of the total density environments described here [5].

The existence of graded population scale responses across two continuously varying environmental inputs (density, genotypic composition) raises the question of why use a 
graded response? Is there an evolutionary rationale for a graded response, or is a graded increase simply the 'best approximation' of a threshold response, given a simple system working under genetic constraints. Existing evolutionary theory suggest that graded investment reaction norms can be adaptive, under a range of distinct scenarios [29,30]. In the specific context of quorum-sensing bacteria, evolutionary theory suggests that population-scale responses to increasing density should depend critically on the shape of the cost and benefit functions of increasing cooperative investments. Specifically, a graded response is predicted to be the optimal strategy if the benefit function is decelerating and costs are linear [31].

To further consider the functional context of the graded reaction norms, we turn to the single cell scale data, which reveals how the graded population response is built from the contributions of individual cells. In agreement with previous work [4,12-16], we find cell-scale heterogeneity, but provide finer resolution and observe heterogeneity in both ON/OFF state and the intensity of the ON state. Our results further illustrate that both the proportion $\mathrm{ON}$ and the intensity of the $\mathrm{ON}$ state increase smoothly with increases in stationary density (Fig 3-5). Together these results illustrate that the graded populationscale response is built from the combination of two graded cellular responses (OFF/ON state, and intensity of $\mathrm{ON}$ ) to changes in density. To assess the relative contribution of these two components, we re-analyzed the data fixing one component and allowing the other to vary (Fig S3). This analysis demonstrates that combining variation in both 'onness' (proportion ON) and intensity of the ON state leads to a steeper reaction-norm than either component alone. In principle, the use of the two components to build the population-scale reaction norm can make a more tunable system; changes in the reaction norm can follow from changes to either the intensity responsiveness, the on-state responsiveness or both.

Our single cell data illustrates that even at high densities $(\mathrm{OD} 600=0.74, \mathrm{CFU}=$ $2.02 \times 10^{9}$ ) a fraction of cells continue to be non-responsive (Fig 4B, max observed proportion non-responsive of 0.06), but still viable (Table S1, max observed non-viable of 0.0176). Persistent heterogeneity of QS response on single cell scale has been reported across multiple studies and model organisms, implicating multiple mechanisms including receptor copy number limitation [12]. Some cells may lack sufficient LasR to respond to a quorum, regardless of the signal concentration. LasR homologs such as LuxR can support a large range of copy numbers [12,32], so LasR insufficiency could be an inevitable result of stochasticity in gene expression. We recognize that our experimental manipulation of stationary density via controlling carbon limitation potentially had other impacts on cellular physiology and therefore QS response. However, we found that controlling density via carbon limitation had little impact on growth rate between environments (Fig S1). We only observed a significant growth rate difference between the lowest carbon and the other treatments $(\mathrm{p}<0.0001)$, and no impact on the proportion of viable cells (Table $\mathrm{S} 1$ ). Apart from growth rate, we recognize the confound that cells will have undergone different numbers of generations. Indeed, Teng. et al have shown that partitioning of LuxR, a LasR homolog, is slightly asymmetric and can generate heterogeneity in responsiveness to signal [32]. 
In summary, introducing population and individual-scale reaction norms to the study of quorum-sensing provides a platform to characterize and compare the functional characteristics of QS systems, and also to place QS within the broader analysis of phenotypic plasticity $[27,28,30]$. Our results provide a finely resolved mapping of the QS reaction norm to environmental density in PAO1, on both the collective and single-cell scale. On the population scale we see a graded linear response across a broad range of cellular densities $\left(1 \times 10^{8}\right.$ cells $/ \mathrm{ml}$ to $2 \times 10^{9}$ cells $\left./ \mathrm{ml}\right)$, and we further resolve this linear response to be the product of two component cellular reaction norms: the likelihood of being responsive and the intensity of response. In an infection context, our results indicate that there is no hard threshold separating sub-quorate 'stealth' mode and a quorate 'attack' mode [33]. One implication is that attempts to control virulence and biofilm expression in medicine and industry via QS inhibition could have greater impacts across a spectrum of population densities. In this applied context, it is important to assess the generality of our results and ask, how do QS reaction-norms vary across strains and species of QS bacteria? How do they vary across environments? More broadly, our work undermines the threshold concept of a 'quorum', instead placing QS bacteria in the graded world of reaction norms.

\section{MATERIALS AND METHODS}

Bacterial Strains and Growth Conditions. The bacterial strains used in this study were $P$. aeruginosa $\mathrm{PAO} 1$ containing the las $B-G F P$ quorum sensing reporter [34] and $P$. aeruginosa PAO1 AlasI/ArhlI lasB-GFP, which is deficient in the production of QS signal molecules. Overnight cultures were grown in Luria-Bertani (LB) broth, supplemented with $100 \mathrm{ug} / \mathrm{ml}$ gentamicin to maintain the pMHLB plasmid, with shaking at $37^{\circ} \mathrm{C}$. Experiments were conducted in lightly buffered (50uM MOPS) minimal defined media composed of an autoclaved basal salts solution $\left(\mathrm{Na}_{2} \mathrm{HPO}_{4}, 6.8 \mathrm{gL}^{-1}\right.$; $\left.\mathrm{KH}_{2} \mathrm{PO}_{4}, 3.0 \mathrm{gL}^{-1} ; \mathrm{NaCl}, 0.5 \mathrm{gL}^{-1}\right)$ and filter-sterilized $1 \mathrm{M}$ supplement stock solutions of $\mathrm{NH}_{4} \mathrm{Cl}, \mathrm{CaCl}_{2}$, and $\mathrm{MgSO}_{4} 7 \mathrm{H}_{2}$ with casamino acids (CAA) as the carbon source.

Controlling Stationary Culture Density. We manipulated density by controlling the limiting resource in the media, carbon, allowing us to tune the carrying capacity of each treatment (Fig S1). To cover a variety of densities, we generated a CAA range between $0.05 \%$ and $0.25 \%$ via dilutions of a $0.5 \%$ CAA minimal media stock for a total of 17 different stationary phase densities. This produced a range of densities environments from $1.18 \times 10^{8}$ cells $/ \mathrm{ml}$ to $2.02 \times 10^{9}$ cells $/ \mathrm{ml}$. Overnight cultures were grown in LB gentamicin $100 \mathrm{ug} / \mathrm{ml}$ and centrifuged at $8,500 \mathrm{x} \mathrm{g}$ for 2 minutes. The cells were then washed twice and resuspended in carbonless minimal media and then adjusted to 0.05 OD600. Aliquots of the various CAA treatments were inoculated with the appropriate strains at a concentration of $10 \mathrm{ul} / \mathrm{ml}$. Then, $200 \mathrm{uL}$ of each sample was added to a 96well microplate and blank minimal media was used as a negative control. Plates were incubated with continuous shaking at $37^{\circ} \mathrm{C}$ in a Hidex Sense plate reader and growth curves were generated by absorbance readings taken at 30-min intervals for a period of $18 \mathrm{hrs}$. 
Measuring QS Response. To measure population response, we performed growth-curve experiments as previously described using PAO1 lasB-GFP, additionally taking fluorescence readings at 30-min intervals. Readings at end exponential phase were extracted and plotted as population level response. Controls were done with the QS signal deficient mutant PAO1 DlasI/ArhlI lasB-GFP.

To measure individual response, we performed growth-curve experiments as previously described, but removed samples for microscopy as they reached end exponential phase. A small aliquot (5ul) was immediately added to a $1.5 \%$ agarose pad to immobilize cells and imaged in the dark on a Nikon Eclipse TI inverted microscope at 20x magnification. Both bright field and green fluorescence (20\% Lumencor light engine power, 200ms exposure, and 64x gain- sufficient for imaging of low fluorescent cells without saturating pixel intensity) channels were captured. Between 5,000 and 15,000 individual cells were captured for each sample. Aliquots were diluted with carbonless minimal media when required to ensure an even distribution of cells. Images were imported into ImageJ, cells were identified on bright field images, and an ROI (region of interest) was generated for each individual cell. These ROIs were then overlaid onto the corresponding fluorescent image, and pixel intensity was then measured as a proxy for level of QS response. Data shown has normalized pixel intensity from 0-255 to a 0-1 scale. This data was then analyzed in $\mathrm{R}$ via the mixtools package [35], which fits gaussian distributions to experimental data. While fitting gaussian distributions allows a non-zero probability of negative values, this is not a prevalent issue due to the minimal negative counts imposed on the data. As our data is bimodal, this results in two curves generated per sample, an $\mathrm{OFF}$ and an $\mathrm{ON}$ distribution. The package also allows for quantification of mixing proportions $(\lambda)$ and mean $(\mu)$ for each distribution. Population level response can also be summarized as the sum of each individual's pixel intensity divided by total number of observed individuals. The trend of both the population microplate data and averaged microscope data agreed, so the latter is provided in this paper.

\section{REFERENCES}

1. Rutherford ST, Bassler BL. Bacterial quorum sensing: Its role in virulence and possibilities for its control. Vol. 2, Cold Spring Harbor Perspectives in Medicine. Cold Spring Harbor Laboratory Press; 2012.

2. Whiteley M, Diggle SP, Greenberg EP. Progress in and promise of bacterial quorum sensing research. Vol. 551, Nature. Nature Publishing Group; 2017. p. 313-20.

3. Hense BA, Kuttler C, Müller J, Rothballer M, Hartmann A, Kreft JU. Does efficiency sensing unify diffusion and quorum sensing? Nat Rev Microbiol. 2007 Mar;5(3):230-9.

4. Boedicker JQ, Vincent ME, Ismagilov RF. Microfluidic Confinement of Single Cells of Bacteria in Small Volumes Initiates High-Density Behavior of Quorum Sensing and Growth and Reveals Its Variability. Angew Chemie Int Ed [Internet]. 2009 Jul 27 [cited 2019 Nov 10];48(32):5908-11. Available from: 
http://doi.wiley.com/10.1002/anie.200901550

5. Darch SE, West SA, Winzer K, Diggle SP. Density-dependent fitness benefits in quorum-sensing bacterial populations. Proc Natl Acad Sci [Internet]. 2012 May 22 [cited 2016 May 19];109(21):8259-63. Available from: http://www.pnas.org/cgi/doi/10.1073/pnas.1118131109

6. Cornforth DM, Popat R, McNally L, Gurney J, Scott-Phillips TC, Ivens A, et al. Combinatorial quorum sensing allows bacteria to resolve their social and physical environment. Proc Natl Acad Sci [Internet]. 2014;111(11):4280-4. Available from: http://www.pnas.org/content/111/11/4280.abstract

7. Allen RC, McNally L, Popat R, Brown SP. Quorum sensing protects bacterial cooperation from exploitation by cheats. ISME J. 2016 Jul 1;10(7):1706-16.

8. Eldar A. Social conflict drives the evolutionary divergence of quorum sensing. Proc Natl Acad Sci U S A. 2011 Aug 16;108(33):13635-40.

9. Even-Tov E, Omer Bendori S, Valastyan J, Ke X, Pollak S, Bareia T, et al. Social Evolution Selects for Redundancy in Bacterial Quorum Sensing. Gore J, editor. PLOS Biol [Internet]. 2016 Feb 29 [cited 2019 Nov 10];14(2):e1002386. Available from: https://dx.plos.org/10.1371/journal.pbio.1002386

10. Özkaya Ö, Balbontín R, Gordo I, Xavier KB. Cheating on Cheaters Stabilizes Cooperation in Pseudomonas aeruginosa. Curr Biol. 2018 Jul 9;28(13):20702080.e6.

11. Chen R, Déziel E, Groleau MC, Schaefer AL, Greenberg EP. Social cheating in a Pseudomonas aeruginosa quorum-sensing variant. Vol. 116, Proceedings of the National Academy of Sciences of the United States of America. National Academy of Sciences; 2019. p. 7021-6.

12. Pradhan BB, Chatterjee S. Reversible non-genetic phenotypic heterogeneity in bacterial quorum sensing. Mol Microbiol. 2014;92(3):557-69.

13. Anetzberger C, Pirch T, Jung K. Heterogeneity in quorum sensing-regulated bioluminescence of Vibrio harveyi. Mol Microbiol [Internet]. 2009 Jul [cited 2019 Nov 10];73(2):267-77. Available from: http://doi.wiley.com/10.1111/j.13652958.2009.06768.x

14. Pérez PD, Hagen SJ. Heterogeneous response to a quorum-sensing signal in the luminescence of individual vibrio fischeri. PLoS One. 2010;5(11).

15. Grote J, Krysciak D, Streit WR. Phenotypic heterogeneity, a phenomenon that may explain why quorum sensing does not always result in truly homogenous cell behavior. Vol. 81, Applied and Environmental Microbiology. American Society for Microbiology; 2015. p. 5280-9.

16. Garmyn D, Gal L, Briandet R, Guilbaud M, Lemaître JP, Hartmann A, et al. Evidence of autoinduction heterogeneity via expression of the agr system of Listeria monocytogenes at the single-cell level. Appl Environ Microbiol. $2011 \mathrm{Sep}$ 1;77(17):6286-9.

17. Miller MB, Bassler BL. Quorum Sensing in Bacteria. Annu Rev Microbiol. 2001 Oct;55(1):165-99.

18. Redfield RJ. Is quorum sensing a side effect of diffusion sensing? Trends Microbiol [Internet]. 2002 Aug [cited 2019 Nov 10];10(8):365-70. Available from: http://www.ncbi.nlm.nih.gov/pubmed/12160634

19. Connell JL, Wessel AK, Parsek MR, Ellington AD, Whiteley M, Shear JB. 
Probing prokaryotic social behaviors with bacterial "lobster traps.” MBio. 2010 Sep;1(4).

20. Fuqua WC, Winans SC, Greenberg EP. Quorum sensing in bacteria: The LuxRLuxI family of cell density- responsive transcriptional regulators. Vol. 176, Journal of Bacteriology. American Society for Microbiology; 1994. p. 269-75.

21. Schluter J, Schoech AP, Foster KR, Mitri S. The Evolution of Quorum Sensing as a Mechanism to Infer Kinship. Locke JCW, editor. PLOS Comput Biol [Internet]. 2016 Apr 27 [cited 2016 May 19];12(4):e1004848. Available from: http://dx.plos.org/10.1371/journal.pcbi.1004848

22. Wang Y, Rattray JB, Thomas SA, Gurney J, Brown SP. In silico bacteria evolve robust cooperation via complex quorum-sensing strategies. bioRxiv [Internet]. 2019 [cited 2019 Nov 12];598508. Available from: https://www.biorxiv.org/content/10.1101/598508v1

23. Goryachev AB. Understanding Bacterial Cell-Cell Communication with Computational Modeling. Chem Rev [Internet]. 2011 Jan 12 [cited 2019 Nov 10];111(1):238-50. Available from: https://pubs.acs.org/doi/10.1021/cr100286z

24. James S, Nilsson P, James G, Kjelleberg S, Fagerström T. Luminescence control in the marine bacterium Vibrio fischeri: An analysis of the dynamics of lux regulation. J Mol Biol. 2000 Mar 3;296(4):1127-37.

25. Woltereck. Weitere experimentelle Untersuchungen uber Artveranderung, speziel uber das Wesen quantitativer Artuntershiede bei Daphniden. Science (80- ). 1910;32(819):344-5.

26. Waddington $\mathrm{CH}$. Canalization of development and the inheritance of acquired characters. Nature. 1942;150(3811):563-5.

27. Schlichting C, Pigliucci M. Phenotypic Evolution: A Reaction Norm Perspective. 1998.

28. Paaby AB, Testa ND. Developmental Plasticity and Evolution. In: Evolutionary Developmental Biology. Springer International Publishing; 2018. p. 1-14.

29. The evolutionary consequences of plasticity in host-pathogen interactions ScienceDirect [Internet]. [cited 2019 Nov 10]. Available from: https:/www.sciencedirect.com/science/article/pii/S0040580905001644?via\%3Dih $\mathrm{ub}$

30. Via S, Gomulkiewicz R, De Jong G, Scheiner SM, Schlichting CD, Van Tienderen PH. Adaptive phenotypic plasticity: consensus and controversy. Vol. 10, Trends in Ecology \& Evolution. 1995. p. 212-7.

31. Heilmann S, Krishna S, Kerr B. Why do bacteria regulate public goods by quorum sensing?-How the shapes of cost and benefit functions determine the form of optimal regulation. Front Microbiol. 2015;6(AUG).

32. Teng SW, Wang Y, Tu KC, Long T, Mehta P, Wingreen NS, et al. Measurement of the copy number of the master quorum-sensing regulator of a bacterial cell. Biophys J. 2010 May 5;98(9):2024-31.

33. Winzer K, Williams P. Quorum sensing and the regulation of virulence gene expression in pathogenic bacteria. Int J Med Microbiol. 2001;291(2):131-43.

34. Hentzer M, Riedel K, Rasmussen TB, Heydorn A, Andersen JB, Parsek MR, et al. Inhibition of quorum sensing in Pseudomonas aeruginosa biofilm bacteria by a halogenated furanone compound. Microbiology. 2002;148(1):87-102. 


\section{Benaglia T, Chauveau D, Hunter DR, Young DS. Mixtools: An R package for} analyzing finite mixture models. J Stat Softw. 2009;32(6):1-29.

\section{SUPPLEMENTARY}
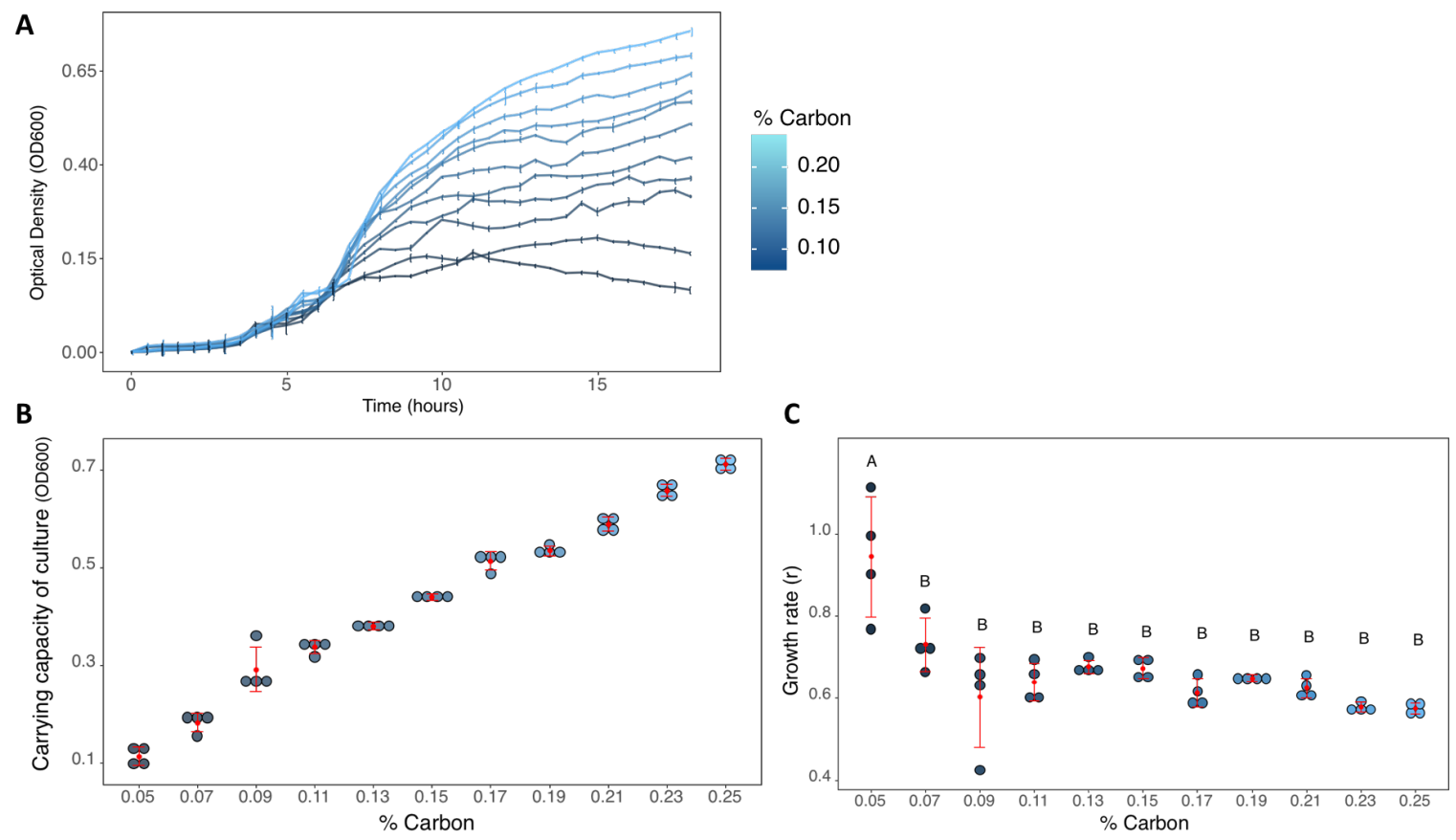

Figure S1. Efficacy of controlling stationary phase density by varying carbon availability. A) Growth curves for samples across different carbon limitations, error bars denote 4 replicates each. Growth data (carrying capacity, $\mathrm{k}$, and growth rate, $\mathrm{r}$ ) was determined by fitting a logistic growth equation to the raw data. B) Carrying capacity of the culture increases with increasing carbon concentration. C) Carbon concentration had minimal impact on growth rate of the bacteria. There was a statistically significant difference between group means as determined by one-way ANOVA $(\mathrm{p}=<0.001)$, but post hoc comparisons using the Tukey-Kramer HSD test indicated that the only significant differences were between $0.05 \%$ carbon and $0.07 \%(\mathrm{p}=0.002)$ and $0.05 \%$ and the rest $(\mathrm{p}<0.0001)$. Even though a significant difference was reported, it is important to note the difficulty of fitting a logistic growth model to the $0.05 \%$ carbon condition due to the similarity in starting density and carry capacity. This is reflected by the increased spread in $0.05 \%$ growth rates. 


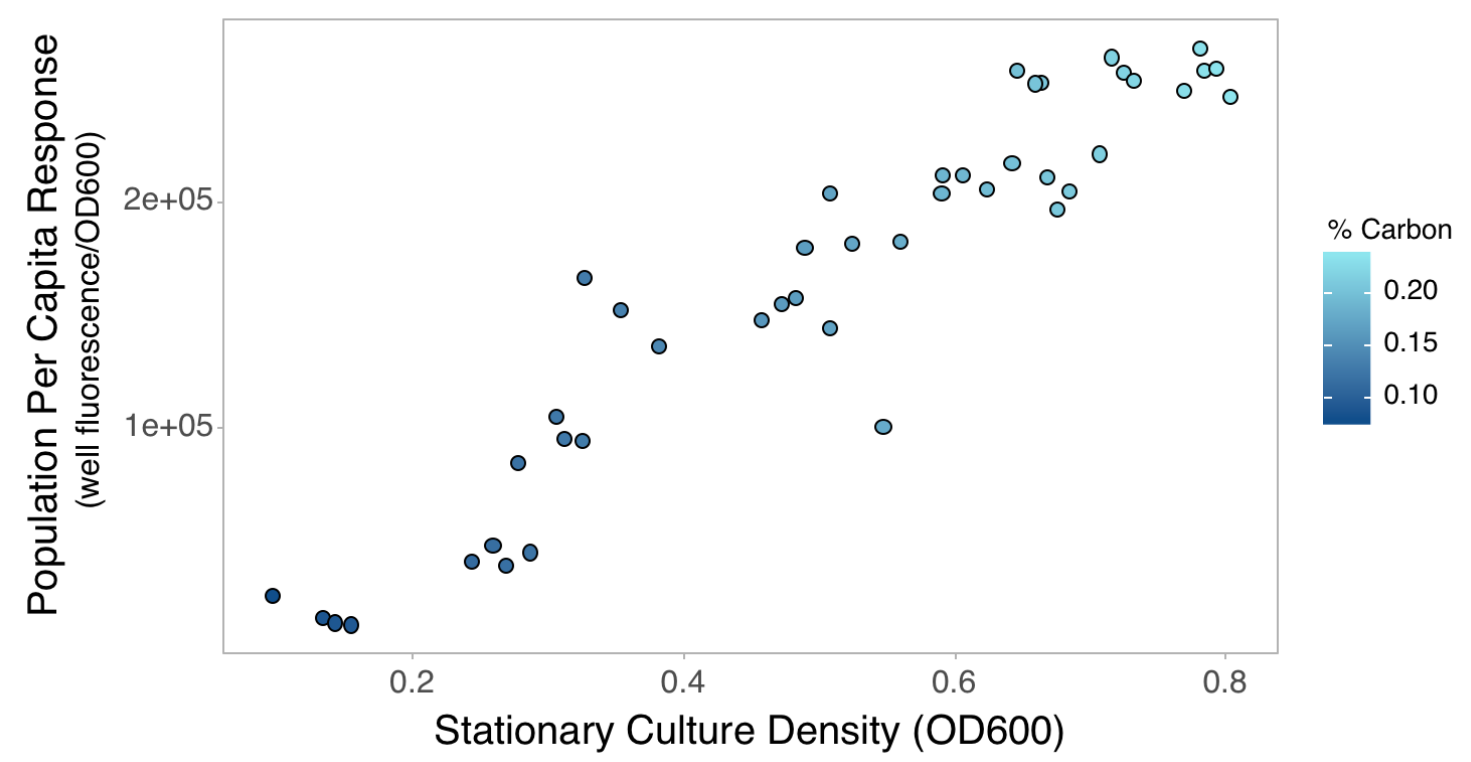

Figure S2. Microplate data. Cultures were grown as mentioned in the method section and fluorescence and OD600 were measured on a Hidex Sense plate reader. Microplate results agree with microscopy results that that population response to increasing cell density is linear and graded. 
A
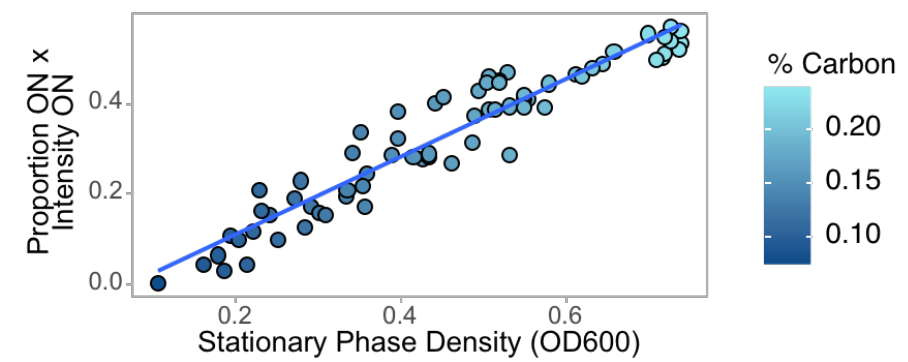

B
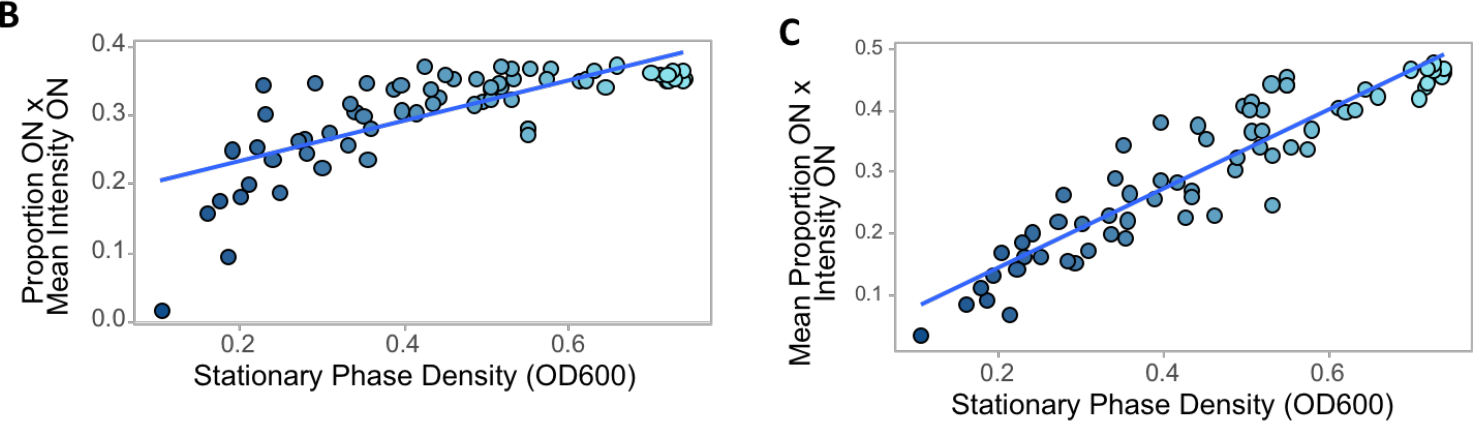

Figure S3. The data were re-analyzed by fixing one component to the mean population value and allowing the other to vary. Linear regressions were performed for each plot to assess the relative contribution of the two observed components (proportion $\mathrm{ON}$ and intensity $\mathrm{ON}$ ). A) Two component reaction norm with both components allowed to vary, $\mathrm{y}=0.857 \mathrm{x}-0.0627, \mathrm{R}^{2}=0.9129$. B) Single component reaction norm with fixed intensity $\mathrm{ON}, \mathrm{y}=0.294 \mathrm{x}+0.172, \mathrm{R}^{2}=0.5518$. C) Single component reaction norm with fixed proportion $\mathrm{ON}, \mathrm{y}=0.638 \mathrm{x}+0.0161, \mathrm{R}^{2}=0.8526$. 


\begin{tabular}{|c|c|}
\hline $\begin{array}{l}\text { Stationary } \\
\text { Culture } \\
\text { Density }\end{array}$ & $\begin{array}{l}\text { Proportion } \\
\text { Non-Viable } \\
\text { Cells }\end{array}$ \\
\hline 0.138 & 0.00742 \\
\hline 0.169 & 0.0145 \\
\hline 0.176 & 0.0105 \\
\hline 0.238 & 0.0103 \\
\hline 0.253 & 0.00379 \\
\hline 0.312 & 0.00239 \\
\hline 0.331 & 0.00166 \\
\hline 0.339 & 0.00383 \\
\hline 0.383 & 0.00418 \\
\hline 0.392 & 0.0176 \\
\hline 0.403 & 0.0174 \\
\hline 0.403 & 0.00812 \\
\hline 0.412 & 0.000637 \\
\hline 0.415 & 0.00305 \\
\hline 0.427 & 0.00343 \\
\hline 0.47 & 0.00181 \\
\hline 0.614 & 0.00149 \\
\hline
\end{tabular}

Table S1. Cultures were grown as mentioned in the method section and then stained for 5 minutes with propidium iodide (PI) at a 1:100 concentration to determine cell viability. PI is a common red-fluorescent intercalating agent that only binds to DNA of membrane-compromised bacteria. The average reported nonviable proportion is $0.00659(+/-0.00561)$ and proportions are un-correlated with density (linear regression, F statistic: 2.153 on 1 and 15 DF, p-value: 0.1629 ). 